\title{
Water vapor permeability of building materials: improved analysis of dry cup experiment
}

\author{
Thibaut Colinart ${ }^{\mathrm{a}^{*}}$, Patrick Glouannec ${ }^{\mathrm{a}}$ \\ a Univ Bretagne Sud, UMR CNRS 6027, IRDL, 56100 Lorient, France
}

\begin{abstract}
Water vapor permeability of building materials is usually measured using dry cup experiment according to the ISO 12572 standard. For this test, suitable adsorbing desiccant should be used to provide stable low vapor pressure conditions within the cup. However, previous measurements on permeable materials have shown that boundary conditions within the cup are not equal to $0 \% \mathrm{RH}$ and not constant over time. The aim of this paper is to extend this finding on other less permeable building materials.

Peer-review under the responsibility of the organizing committee of the ICMB21.
\end{abstract}

Keywords: Water vapor permeability; Dry cup; OSB; Hollow Concrete Block; Rainscreen membrane; AAC; Gypsum;

\section{Introduction}

Water vapor permeability is one of the required properties when performing hygrothermal simulation of building envelopes. This property is determined by means of cup test by most of building scientists. As detailed in the latest version of the standard ISO 12572 [1], this test consists in measuring under steady state and isothermal condition the moisture flow due to relative humidity gradient across a sample sealed on a cup containing desiccant or saturated salt solutions. Despite the simplicity of the methods, numerous round robin tests [2-4] showed large inter-laboratory discrepancies. Among the possible factors explaining these discrepancies, inadequate control of ambient conditions was found to be of high importance for permeable materials. Indeed, a previous set of instrumented dry cup experiments performed on wood fiber insulation has shown that relative humidity measured within the cup is not constant over time whatever the desiccant used [5]. Consequently, steady state is not necessary reached, avoiding a proper estimation of water vapor permeability as specified in the standard ISO 12572. Furthermore, the measured relative humidity is not equal to $0 \% \mathrm{RH}$, leading to errors up to $300 \%$ in the calculated water vapor diffusion resistance factor. The objective of this paper is to extend these findings on less permeable building materials.

\section{Materials and methods}

Numerous building materials (ranged from the less to the more permeable in terms of Sd-value) are investigated: Hollow Concrete Blocks (HCB), OSB boards (OSB1 \& 2), Rainscreen membrane (Memb.), Aerated Autoclaved Concrete (AAC) and Gypsum board (Gyp.). All samples have surface of $100 \times 100 \mathrm{~mm}^{2}$, while the thickness ranges from 0.6 to $20 \mathrm{~mm}$. Prior to testing, samples are dried according to EN 13171 standard and then conditioned at $23{ }^{\circ} \mathrm{C}$ and $50 \%$ RH. They are then sealed to cups containing desiccant. Here, the cups with dimensions $100 \times 100 \times 60 \mathrm{~mm}^{3}$ are made of PE and a vapor-tight aluminum tape was used to seal the sides of the sample with the side of the cup. Three desiccants are tested in this work: new silica gel (regenerated one time at $120{ }^{\circ} \mathrm{C}$ ), calcium chloride $\mathrm{CaCl}_{2}$ powder (not regenerated prior to the experiment), saturated solution of potassium hydroxide $\mathrm{KOH}$. Whatever the desiccant, the thickness of air layer between specimen and desiccant is always in the range $17 \pm 3 \mathrm{~mm}$. Temperature and relative humidity in this air layer are monitored with an accuracy of $2 \% \mathrm{RH}$ and $0.5{ }^{\circ} \mathrm{C}$ with HygroPuce (Waranet, Auch, France). Assemblies are placed in a climatic chamber (Memmert HPP 108, Schwabach, Germany) at $23{ }^{\circ} \mathrm{C}$ and $50 \% \mathrm{RH}$. Air velocity measured above the cup with a hot-wire anemometer is around $0.15 \pm 0.05 \mathrm{~m} . \mathrm{s}^{-1}$. Cups are removed punctually from the chamber to be weighed using a balance with a precision of $0.01 \mathrm{~g}$ (Adventurer Pro AV4102C, Ohaus Corporation, Pine Brook, NJ, USA). The minimal weighing interval is set according to the annex I of the standard [1] to get results with an accuracy of $5 \%$. The measurements are performed until water vapor flow rate $g$ reaches steady state (as specified in section 8.1 of the standard [1]) or until the assembly has gained more than $1.5 \mathrm{~g}$ per $25 \mathrm{ml}$ of desiccant in the cup. For this set-up, it corresponds to a mass gain of about $2.5 \mathrm{~kg} \cdot \mathrm{m}^{-2}$. Here, experiments run for at least 15 days.

* Corresponding author. +33297874517. thibaut.colinart@univ-ubs.fr

(C) The Author(s). This is an open access article distributed under the terms of the Creative Commons Attribution License (CC BY) 4.0 https:// creativecommons.org/licenses/by/4.0/, which permits unrestricted use, distribution and reproduction in any medium, provided the original author and source are credited. DOI: 10.14293/ICMB210017 


\section{ICMB21}

\section{Results and discussion}

Table 1 gathers the minimal relative humidity measured within the air layer $\left(R H_{\min }\right)$ and its difference to the relative humidity measured after 15 days $(\Delta R H)$. Globally, we note that $R H_{\min }$ differs from theoretical relative humidity for each dessiccant $(0 \% \mathrm{RH}$ for silica gel and $\mathrm{CaCl}_{2}$ and $8.5 \% \mathrm{RH}$ for $\mathrm{KOH}$ ). It means that moisture provided by exterior air and sample is not instantaneously and totally adsorbed by the desiccant. Furthermore, we observe that relative humidity varies over time within the air layer (up to $18 \% \mathrm{RH}$ ) when solid desiccants are used: desiccant adsorption capacity is reduced as experiment proceeds. On the other hand, $\triangle \mathrm{RH}$ do not exceed $1.5 \%$ for $\mathrm{KOH}$ saturated solutions, meaning that stable conditions can be reached when oversaturated solutions are used. These observations are in agreement with previous results obtained on wood fiber insulation [5], but they are less pronounced for less permeable materials like HCB or OSB1.

Table 1. minimal relative humidity measured within the air layer $\left(R H_{\min }\right)$ and its difference to the relative humidity measured after 15 days $(\Delta R H)$.

\begin{tabular}{|l|c|c|c|c|c|c|c|c|c|c|c|c|}
\hline \multicolumn{1}{|c|}{ Material } & \multicolumn{2}{|c|}{ HCB } & \multicolumn{2}{c|}{ Memb. } & \multicolumn{2}{c|}{ OSB1 } & \multicolumn{2}{c|}{ OSB2 } & \multicolumn{2}{c|}{ AAC } & \multicolumn{2}{c|}{ Gyp. } \\
\hline & $R H_{\min }$ & $\Delta R H$ & $R H_{\min }$ & $\Delta R H$ & $R H_{\min }$ & $\Delta R H$ & $R H_{\min }$ & $\Delta R H$ & $R H_{\min }$ & $\Delta R H$ & $R H_{\min }$ & $\Delta R H$ \\
\hline Silica Gel & 4.57 & 6.00 & 4.18 & 13.73 & 10.58 & 2.01 & 12.59 & 5.59 & 16.61 & 12.10 & 18.96 & 18.17 \\
\hline $\mathrm{CaCl}_{2}$ & 5.77 & 3.29 & 10.12 & 5.93 & 5.96 & 3.82 & 11.00 & 3.45 & 6.56 & 8.36 & 12.49 & 14.84 \\
\hline $\mathrm{KOH}$ & 12.20 & 1.41 & 17.79 & 0.96 & 10.29 & 0.97 & 12.77 & 0.71 & 25.01 & 0.76 & 27.90 & 1.46 \\
\hline
\end{tabular}

Despite the relative humidity variations within the air layer, steady state (as defined in the standard ISO 12572 [1]) could be reached for all experiments, except for Rainscreen membrane and Gypsum board tested with Silica Gel. Water vapor diffusion resistance factor $\mu$ is then calculated by considering air surface resistances using either a theoretical or the measured relative humidity within the air layer. Results for OSB1 and Gypsum board are plotted in Figure 1. Using theoretical value for relative humidity leads to large discrepancies between the three desiccants. These differences are reduced when using the measured relative humidity. Furthermore, the calculated water vapor diffusion resistance factors are reduced by $5 \%$ to $20 \%$ for OSB 1 (less permeable material) and by $87 \%$ to $280 \%$ for Gypsym board (more permeable material). Such differences are in agreement with previous finding on wood fiber insulation [5] and confirm that care should be taken when measuring the water vapor diffusion resistance factor of permeable building materials with dry cup experiments.

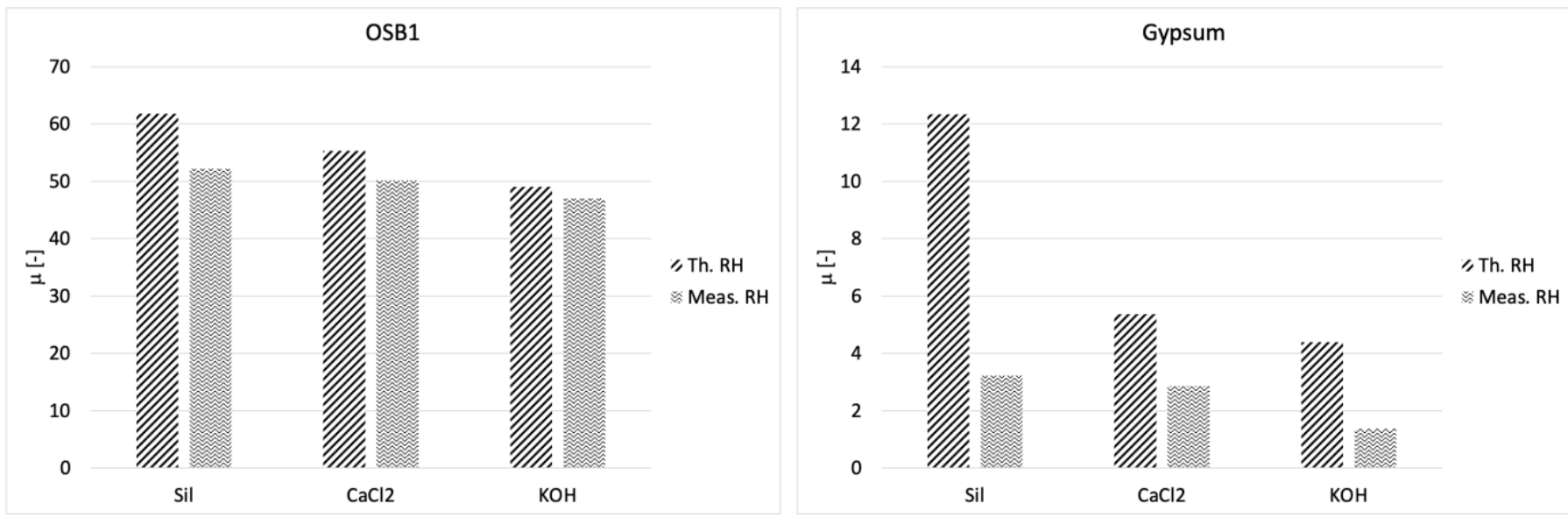

Figure 1. Water vapor diffusion resistance factor $\mu$ of OSB1 (a) and Gypsum (b) calculated with theoretical or measured relative humidity within the air layer for three desiccants.

\section{References}

[1] ISO 12572 (2016). Hygrothermal performance of building materials and products - Determination of water vapour transmission properties-Cup Method.

[2] Feng, C., Guimarães, A.S., Ramos, N., Sun, L., Gawin, D., Konca, P., Zhao, J., Grunewald, J., Hansen, K.K., Hall, C., Fredriksson, M., Pavlík, Z., Janssen, H. (2020). Hygric properties of porous building materials (VI): A round robin campaign. Building and environment, 185,107242

[3] Roels, S., Talukdar, P., James, C., Simonson, C.J.. (2010). Reliability of material data measurements for hygroscopic buffering. International Journal of Heat and Mass Transfer, 53, 5355-5363.

[4] Roels, S., Carmeliet, J., Hens, H., Adan, O., Brocken, H., Cerny, R., Pavlík, Z., Hall, C., Kumaran, K., Pel, L., Plagge, R. (2004). Interlaboratory comparison of hygric properties of porous building materials. Journal of thermal envelope and building science, 27(4), 307-325.

[5] Colinart, T., \& Glouannec, P. (2020). On the importance of desiccant during the determination of water vapor permeability of permeable insulation material. In E3S Web of Conferences (Vol. 172, p. 14003). EDP Sciences. 